\title{
Clinical Rating Scale for Pantothenate Kinase-Associated Neurodegeneration: A Pilot Study
}

\author{
Alejandra Darling, MD, ${ }^{1}$ Cristina Tello, $\mathrm{PhD},{ }^{2}$ María Josep Martí, MD, $\mathrm{PhD},{ }^{3}$ Cristina Garrido, $\mathrm{MD},{ }^{4}$ \\ Sergio Aguilera-Albesa, MD, PhD, ${ }^{5}$ Miguel Tomás Vila, MD, ${ }^{6}$ Itziar Gastón, MD, ${ }^{7}$ Marcos Madruga, MD, ${ }^{8}$ \\ Luis González Gutiérrez, MD, ${ }^{9}$ Julio Ramos Lizana, MD, ${ }^{10}$ Montserrat Pujol, MD, ${ }^{11}$ Tania Gavilán Iglesias, MD, ${ }^{12}$ Kylee Tustin, ${ }^{13}$ \\ Jean Pierre Lin, MD, PhD, ${ }^{13}$ Giovanna Zorzi, MD, PhD, ${ }^{14}$ Nardo Nardocci, MD, PhD, ${ }^{14}$ Loreto Martorell, PhD, ${ }^{15}$ \\ Gustavo Lorenzo Sanz, MD, ${ }^{16}$ Fuencisla Gutiérrez, MD, ${ }^{17}$ Pedro J. García, MD, ${ }^{18}$ Lidia Vela, MD, ${ }^{19}$ \\ Carlos Hernández Lahoz, MD, ${ }^{20}$ Juan Darío Ortigoza Escobar, MD, ${ }^{1}$ Laura Martí Sánchez, ${ }^{1}$ Fradique Moreira, MD (DiD, ${ }^{21}$ \\ Miguel Coelho, MD, ${ }^{22}$ Leonor Correia Guedes, ${ }^{23}$ Ana Castro Caldas, MD, ${ }^{24}$ Joaquim Ferreira, MD, ${ }^{22,23}$ Paula Pires, MD, ${ }^{24}$ \\ Cristina Costa, MD, ${ }^{25}$ Paulo Rego, MD, ${ }^{26}$ Marina Magalhães, MD, ${ }^{27}$ María Stamelou, MD, ${ }^{28,29}$ Daniel Cuadras Pallejà, MD, ${ }^{30}$ \\ Carmen Rodríguez-Blazquez, PhD, ${ }^{31}$ Pablo Martínez-Martín, MD, PhD, ${ }^{31}$ Vincenzo Lupo, PhD, ${ }^{2}$ Leonidas Stefanis, MD, ${ }^{28}$ \\ Roser Pons, MD, ${ }^{32}$ Carmen Espinós, PhD, ${ }^{2}$ Teresa Temudo, MD, PhD, ${ }^{4}$ and Belén Pérez Dueñas, MD, PhD ${ }^{1,33 *}$
}

${ }^{1}$ Unit of Pediatric Movement Disorders, Hospital Sant Joan de Déu, Barcelona, Spain

${ }^{2}$ Unit of Genetics and Genomics of Neuromuscular and Neurodegenerative Disorders, Centro de Investigación Príncipe Felipe, Valencia, Spain ${ }^{3}$ Neurology Department, Hospital Clínic de Barcelona, Institut d'Investigacions Biomediques IDIBAPS. Barcelona, Catalonia,

Centro de Investigación Biomédica en Red-Neurodegenerativas (CIBERNED), Madrid, Spain

${ }^{4}$ Pediatric Neurology Department, Centro Materno-Infantil Centro Hospitalario do Porto, Porto, Portugal

${ }^{5}$ Pediatric Neurology Department, Complejo Hospitalario de Navarra, Navarrabiomed, Pamplona, Spain

${ }^{6}$ Pediatric Neurology Department, Hospital Universitario Politécnico La Fe, Valencia, Spain

${ }^{7}$ Neurology Department, Complejo Hospitalario de Navarra, Pamplona, Spain

${ }^{8}$ Pediatric Neurology Department, Hospital Universitario Virgen del Rocío, Sevilla, Spain

${ }^{9}$ Pediatric Neurology Department, Hospital Infantil Universitario Niño Jesús, Madrid, Spain

${ }^{10}$ Pediatric Neurology Department, Hospital Torrecárdenas, Almería, Spain

${ }^{11}$ Neurology Department, Hospital Santa María, Lérida, Spain

${ }^{12}$ Neurology Department, Hospital de Mérida, Extremadura, Spain

${ }^{13}$ Children's Neurosciences, Evelina London Children's Hospital, Guy's and St Thomas' NHS Foundation Trust, London, United Kingdom

${ }^{14}$ Department of Pediatric Neuroscience, Fondazione IRCCS "C. Besta", Milano, Italy

${ }^{15}$ Molecular Genetics Department, Hospital Sant Joan de Déu, Barcelona. CIBERER, Instituto de Salud Carlos III, Madrid, Spain

${ }^{16}$ Pediatric Neurology Department, Hospital Ramón y Cajal, Madrid, Spain

${ }^{17}$ Neurology Department, Complejo Asistencial Universitario de Palencia, Palencia, Spain

${ }^{18}$ Neurology Department, Fundación Jiménez Díaz, Madrid, Spain

${ }^{19}$ Neurology Department, Hospital de Alcorcón, Madrid, Spain

${ }^{20}$ Neurology Department, Hospital Central de Asturias, Oviedo, Spain

${ }^{21}$ Neurology Department, Centro Hospitalar e Universitário de Coimbra, Coimbra, Portugal

${ }^{22}$ Clinical Pharmacology Unit, Instituto de Medicina Molecular and Department of Neurosciences, Service of Neurology, Hospital Santa Maria, Lisboa, Portugal

${ }^{23}$ Laboratory of Clinical Pharmacology and Therapeutics, Lisbon Faculty of Medicine, Lisbon, Portual

${ }^{24}$ Neurology Department, Hospital de Santo Espirito, Ilha Terceira, Portugal

${ }^{25}$ Neurology Department, Hospital Fernando Fonseca, Lisboa, Portugal

${ }^{26}$ Pediatric Department, Hospital Central de Funchal, Funchal, Portugal

${ }^{27}$ Neurology Department, Centro Hospitalar Porto, Porto, Portugal

${ }^{28}$ Second Department of Neurology, Medical School, National and Kapodistrian University of Athens, Athens, Greece

${ }^{29}$ Parkinson's Disease and other Movement Disorders Department, HYGEIA Hospital, Athens, Greece

${ }^{30}$ Statistical Department, Sant Joan de Déu Fundation, Barcelona, Spain

*Correspondence to: Belén Pérez-Dueñas. Child Neurology Department, Hospital Sant Joan de Déu, Passeig Sant Joan de Déu, 2, 08950, Esplugues, Barcelona, Spain; bperez@hsjdbcn.org

Relevant conflicts of interests/financial disclosures: Authors report no disclosures.

Funding agencies: This work was supported by Fundació La Marató TV3 (grants 20143130 [to B.P.D.] and 20143131 [to CE]), "Plan Nacional de I+D+I and Instituto de Salud Carlos III- Subdirección General de
Evaluación y Fomento de la Investigación Sanitaria" (grants FIS PI15/ 00287 and Rio Hortega CM16/00084 [to J.D.O.E.), the European Social Fund (ESF), and Retrophin, Inc. (grant 049 [to B.P.D.]).

Received: 20 December 2016; Revised: 22 June 2017; Accepted: 26 June 2017

Published online 28 August 2017 in Wiley Online Library (wileyonlinelibrary.com). DOI: 10.1002/mds.27129 
${ }^{31}$ National Center of Epidemiology and CIBERNED, Institute of Health Carlos III, Madrid, Spain

${ }^{32}$ Pediatric Neurology Unit, First Department of Pediatrics, Medical School, National and Kapodistrian University of Athens, Hospital Agia Sofía, Athens, Greece

${ }^{33}$ CIBERER, Instituto de Salud Carlos III, Madrid, Spain

\begin{abstract}
Background: Pantothenate kinaseassociated neurodegeneration is a progressive neurological disorder occurring in both childhood and adulthood. The objective of this study was to design and pilot-test a disease-specific clinical rating scale for the assessment of patients with pantothenate kinaseassociated neurodegeneration.

Methods: In this international cross-sectional study, patients were examined at the referral centers following a standardized protocol. The motor examination was filmed, allowing 3 independent specialists in movement disorders to analyze 28 patients for interrater reliability assessment. The scale included 34 items (maximal score, 135) encompassing 6 subscales for cognition, behavior, disability, parkinsonism, dystonia, and other neurological signs.

Results: Forty-seven genetically confirmed patients (30 \pm 17 years; range, 6-77 years) were examined with the scale (mean score, $62 \pm 21$; range, 20-106). Dystonia with prominent cranial involvement and atypical parkinsonian features were present in all patients. Other common signs were cognitive impairment, psychiatric features, and slow and hypometric saccades. Dystonia,
\end{abstract}

parkinsonism, and other neurological features had a moderate to strong correlation with disability. The scale showed good internal consistency for the total scale (Cronbach's $\alpha=0.87$ ). On interrater analysis, weighted kappa values (0.30-0.93) showed substantial or excellent agreement in $85 \%$ of the items. The scale also discriminated a subgroup of homozygous c.1583C>T patients with lower scores, supporting construct validity for the scale.

Conclusions: The proposed scale seems to be a reliable and valid instrument for the assessment of pediatric and adult patients with pantothenate kinaseassociated neurodegeneration. Additional validation studies with a larger sample size will be required to confirm the present results and to complete the scale validation testing. (c) 2017 International Parkinson and Movement Disorder Society

Key Words: pantothenate kinase-associated neurodegeneration; PKAN; clinical rating scale; neurodegeneration with brain iron accumulation; dystonia parkinsonism
Pantothenate kinase-associated neurodegeneration (PKAN) from mutations in the PANK2 gene is the most common subtype of neurodegeneration with brain iron accumulation disorders. ${ }^{1,2}$ Internationally validated rating scales for dystonia, parkinsonism, and ataxia $^{3-6}$ have been used in PKAN series to capture clinical improvement after deep brain stimulation ${ }^{7-10}$ or iron-chelating therapies. ${ }^{11-13}$ However, PKAN patients show an overlapping dystonia-parkinsonism syndrome $^{14}$ that cannot be easily differentiated, as well as other motor abnormalities (ie, chorea, pyramidal signs, or abnormal ocular movements) and neuropsychiatric disturbances (cognitive decline, behavioral abnormalities) that are not always recorded in the above-mentioned scales. Consequently, there is a lack of specific validated instruments to measure the severity and the rate of disease progression in a multidimensional and comparable manner.

In this study, we aimed to design and pilot-test a disease-specific clinical rating scale named the PKAN Disease Rating Scale (PKAN-DRS). The following clinimetric attributes were analyzed: acceptability, internal consistency, reproducibility, and construct validity. Moreover, the study allowed an accurate assessment of the broad neurological spectrum and the consequent functional impairment associated with PKAN.

\section{Materials and Methods}

\section{Design and Participants}

Cross-sectional multicenter study of 47 PKAN patients recruited at 24 centers in Europe from January 2014 to June 2016. Children and adults with genetically confirmed PKAN were included. Participants were assessed following a standardized protocol at the referral centers or at the coordinator center (Hospital Sant Joan de Déu, Barcelona), some of them during the first neurodegeneration with brain iron accumulation Spanish family meeting that took place on November 2015 (http://www.guiametabolica.org/ noticia/primer-encuentro-a sociacion-enach-espanola). In cases from Greece, Portugal, and Spain, expert neurologists from the collaborating centers performed a direct translation without back-translation from the original English version of the scale. 
All participants or their legal guardians provided informed consent for their participation in the study. All procedures followed the Helsinki Declaration of 1975 , as revised in 2000 . The study was approved by the respective hospital ethical committees.

\section{Development of the PKAN-Disease Rating Scale}

We designed the PKAN-Disease Rating Scale (PKANDRS) to include the main clinical features of PKAN patients according to the literature review and our personal experience, and used the following internationally validated scales as references: Unified Huntington's Disease Rating Scale, ${ }^{15}$ Movement Disorder SocietyUnified Parkinson's disease Rating Scale (MDSUPDRS), ${ }^{16}$ Unified Multiple System Atrophy Disease Rating Scale (UMSARS), ${ }^{17}$ Burke-Fahn-Marsden Dystonia Rating Scale (BFMDRS), ${ }^{3}$ and Ashworth Scale. ${ }^{18}$ The Movement Disorder Society granted permission to use select items from the MDS-UPDRS for parkinsonism and the UMSARS for ocular motility in the PKANDRS. Also, the Huntington Study Group granted permission to include the UHDRS Behavioral Examination in the PKAN-DRS. The expert clinicians involved in the design of the scale were responsible for the item selection. We decided to give more weight to the motor examination evaluation, which provided objective data for the assessment process, in contrast to the questionnaires addressing neuropsychiatric symptoms, which were completed by caregivers in most cases because of the severe speech difficulties of PKAN patients. The rationale weight of the subscales (SCs) addressing the motor aspects of the disease (dystonia, parkinsonism, and other neurological signs) was calculated according to the phenotype of patients included in our series, who showed a prominent parkinsonism-dystonia syndrome. Because dystonia was more disabling than parkinsonism in our cohort, we decided to assess dystonic signs bilaterally in contrast to parkinsonian signs, which were rated in the most affected limb.

The PKAN-DRS was built as a single scale of 34 items grouped into 6 SCs (Appendix 1) that can be summed to render a total score (maximum score, 135) or be analyzed separately. Each item of the scale was followed by a text describing 5 commonly accepted clinical terms: $0=$ normal, $1=$ slight $($ symptoms $/$ signs with sufficiently low frequency or intensity to cause no impact on function), 2 = mild (symptoms/signs of frequency or intensity sufficient to cause a modest impact on function), $3=$ moderate (symptoms/signs sufficiently frequent or intense to cause considerable impact, but not prevent function), and $4=$ severe (symptoms/signs that prevent function). ${ }^{16}$ Exceptionally, 3 items from the disability score had different maximum ratings (walking, maximum score of 5; vision and school/work, maximum score of 3).
In contrast to this rating system, psychiatric features and ocular motor dysfunction were rated according to the number of symptoms that were identified in the patient.

\section{Questionnaire}

Cognition (SC I), behavior (SC II), and disability (SC III) were scored according to the patient's or caregiver's interview, based on questions regarding the situation of patients in the last month. These SCs contributed $3 \%$ each (4 of 135 ; SC I and II) and $26 \%$ (35 of 135; SC III) to the global scale.

SC III was a modification of the BFM disability scale (BFM-DS), which is based on the patient's or caregiver's report of disability in 7 activities of daily living. We added 2 more items to rate visual function ${ }^{19}$ and the capability of patients to engage at school (children) or at any kind of employment (adults).

\section{Motor Examination}

Parkinsonism (SC IV), dystonia (SC V), and other neurological signs (SC VI) were recorded using a videotaped examination protocol designed for the PKANDRS (Appendix 2). The investigators followed the statement "rate what you see," assuming that overlapping of features in PKAN patients (eg, parkinsonism, dystonia, chorea, or spasticity) may interfere with the assessment of individual items in the motor examination. In situations where it was impossible to test severity, the examiner used the notation "UR," for unable to rate. A brief explanation on how to rate the different items has been included in the PKAN-DRS (Appendix 1).

SC IV included 7 parkinsonian signs that were selected from the Part III motor examination of the MDS-UPDRS, contributing 21\% (28 of 135) to the global scale. We selected finger tapping and leg agility to assess limb bradykinesia, as they have been considered reliable tests in Parkinson's disease and atypical parkinsonisms. ${ }^{17,20-22}$

SC V for dystonia was adapted from the BFM motor scale (BFM-MS). We added 2 items for dystonic eye deviation and jaw/tongue dystonia, based on the specific characteristics of dystonia in PKAN patients. We did not apply the weighting factor (0.5) to the eyes, mouth, and neck, as in the original scale, because these areas are prominently affected in PKAN. Dystonia was rated according to the maximal severity observed during the protocol evaluation, and we did not use the provoking factor of the BFM-MS to prevent poor consistency among raters. ${ }^{6,23}$ Overall, 10 body parts were evaluated, contributing 29\% (40 of $135)$ to the global scale. SC VI was created to include other neurological signs: speech, gait, chorea, spasticity, postural or action tremor, and ocular motor 
dysfunction. This subscale contributed $18 \%$ (24 of 135) to the global scale.

\section{Assessment and Procedure}

The 47 PKAN participants were evaluated on-site with the questionnaire and the videotaped examination protocol. Using the videotaped material, 3 independent senior specialists in movement disorders (rater 1, R.P., child neurologist; rater 2, B.P.D., child neurologist; rater 3, M.J.M., adult neurologist) analyzed 28 patients recruited between January 2014 and November 2015 for interrater reliability assessment. These examiners rated 21 items (SC IV, 6 items; SC V, 10 items; SC VI, 5 items). Rigidity and spasticity were exclusively rated in person; hence, they were excluded from this analysis. Each rater used a report form to record data and to obtain independent assessments. Raters were not allowed to exchange opinions of the evaluation.

\section{Molecular Analyses}

We performed genetic analysis of point mutations by sequencing the codified exons and their flanking intronic regions (NM_153638.2) using the Sanger method. The analysis of large deletions and/or duplications was completed by Multiplex Ligation-Dependent Probe Amplification (SALSA MLPA probemix P120B2; MRC Holland, Amsterdam, The Netherlands).

\section{Statistical Analysis}

All data analyses were conducted using the SPSS version 24.0 statistical package. To describe patient data, we computed means, standard deviations, median, interquartile ranges, and total ranges.

The scale was considered acceptable when missing responses were less than $5 \%,{ }^{24}$ mean scores were near the scale midpoint, floor and ceiling effects were less than $20 \%$, and skewness statistics were between -1 and $+1.25,26$

Internal consistency of the total scale and the SCs was determined by Cronbach's alpha $(\alpha)$, and a value of 0.70 or greater was considered acceptable. ${ }^{27}$ Correlations among the PKAN-DRS I-VI SCs were performed, and an a priori hypothesis was made that SC III would show close correlation with other SCs. Given the diversity of symptoms evaluated in the PKAN-DRS, we expected low or moderate correlations $(<0.60)$ between other domains. ${ }^{28}$ Also, corrected item-total correlations were analyzed. ${ }^{29}$

To determine the interrater reliability, kappa statistics calculating quadratic disagreement weights (weighted kappa $[\mathrm{kw}]$ ) was used to measure the interrater reliability for individual items in all possible pairs of raters. ${ }^{27}$ Moreover, the intraclass correlation coefficient (2 way, random effect [ICC]) with $95 \%$ confidence intervals (CIs) was determined to assess the reproducibility of each subscale and the total scale in the 3 raters simultaneously. ICC values were considered excellent $(>0.90)$, good (0.90-0.75), and poor to moderate $(<0.75) .{ }^{30}$

Spearman's rank correlation coefficients were calculated to test the hypothesis that PKAN-DRS would show strong correlation $\left(r_{S}>0.60\right)^{30}$ with the BFMDRS. Also, we hypothesized that disease severity (measured by the PKAN-DRS total scale and subscales) would correlate negatively with age at onset and positively with disease duration. A multiple linear regression analysis was performed, and results are shown as standardized regression coefficients (beta).

Known-groups validity testing was performed to test the hypothesis that homozygous c. $1583 \mathrm{C}>\mathrm{T}$ patients had a milder phenotype than patients with other PANK2 mutations (Mann-Whitney $U$ test).

$P<0.05$ was considered significant.

\section{Results}

\section{Demographics and Disease-Related Data}

Table 1 shows 47 PKAN patients (mean age at disease onset $\pm \mathrm{SD}, 12.5 \pm 9.5$ years; range, $0.75-51$ years; mean age at assessment $\pm S D, 30 \pm 17$ years; range, 6 77 years) from 41 families were examined with the PKAN-DRS. Disease duration (years of evolution of the disease at the time of assessment) was $18 \pm 14.5$ years (range, 2-61 years).

\section{Clinical Assessment With the PKAN-DRS}

The PKAN-DRS video protocol was completed in a mean time \pm SD of $13.7 \pm 2.4$ minutes (range, 9.716.2 minutes). Mean scores for the 34 items are shown in Table 2 and Figure 1.

\section{Questionnaire}

Twenty-six patients $(56 \%)$ reported some degree of cognitive impairment, and 34 participants $(72 \%)$ reported at least 1 of the following psychiatric symptoms: sadness and mood disorders $(\mathrm{n}=20)$, irritable behavior $(\mathrm{n}=14)$, anxiety $(\mathrm{n}=12)$, obsessions and compulsions $(\mathrm{n}=11)$, disruptive or aggressive behavior $(\mathrm{n}=5)$, suicidal thoughts $(\mathrm{n}=2)$, and hallucinations $(\mathrm{n}=2)$.

Functional impairment (score $\geq 1$ ) was identified on the following motor and nonmotor experiences of daily living: speech $(\mathrm{n}=47)$, walking $(\mathrm{n}=46)$, handwriting $(n=45)$, feeding $(n=42)$, school/work performance $(\mathrm{n}=42)$, dressing $(\mathrm{n}=40)$, hygiene $(\mathrm{n}=$ $39)$, and swallowing $(\mathrm{n}=35)$. Visual impairment was reported in 8 patients.

\section{Motor Examination}

Parkinsonian features were identified in all patients. Some patients could not perform some of the motor tasks because of an overlap between parkinsonism and 
D A R L I N G E T A L

TABLE 1. Demographic data, global PKAN-DRS scores, and PANK2 mutations in the entire cohort of 47 PKAN patients

\begin{tabular}{|c|c|c|c|c|c|c|c|}
\hline Patient & Age at onset & Sex/current age & Ethnicity & Gait & PKAN DRS score & PANK2 allele 1 & PANK2 allele 2 \\
\hline 1 & 10 & $F / 13$ & White European & Independent & 63 & c.747dupC & c. $1475 C>G$ \\
\hline 2 & 13 & $\mathrm{M} / 18$ & Roma Population & Independent & 55 & c. $1583 \mathrm{C}>\mathrm{T}$ & c. $1583 C>T$ \\
\hline 3 & 18 & $F / 22$ & Roma Population & Independent & 38 & c. $1583 C>T$ & c. $1583 C>T$ \\
\hline 4 & 10 & $M / 36$ & Roma Population & Assisted & 75 & c. $1583 \mathrm{C}>\mathrm{T}$ & c. $1583 \mathrm{C}>\mathrm{T}$ \\
\hline 5 & 25 & $\mathrm{M} / 35$ & Roma Population & Independent & 46 & c. $1583 \mathrm{C}>\mathrm{T}$ & c. $1583 \mathrm{C}>\mathrm{T}$ \\
\hline 6 & 34 & $\mathrm{M} / 39$ & White European & NA (38) & 42 & c. $1211 \mathrm{~A}>\mathrm{T}$ & c. $1211 \mathrm{~A}>\mathrm{T}$ \\
\hline 7 & 6 & $\mathrm{M} / 16$ & Roma Population & Independent & 32 & c. $1583 \mathrm{C}>\mathrm{T}$ & c. $1583 \mathrm{C}>\mathrm{T}$ \\
\hline 8 & 9 & $\mathrm{~F} / 41$ & White European & NA (22) & 87 & c. $570 \mathrm{C}>\mathrm{G}$ & c. $790 \mathrm{C}>\mathrm{T}$ \\
\hline 9 & 13 & $\mathrm{~F} / 20$ & White European & NA (18) & 79 & c. $1070 \mathrm{G}>\mathrm{C}$ & c. $790 \mathrm{C}>\mathrm{T}$ \\
\hline 10 & 6 & $\mathrm{M} / 43$ & White European & NA (21) & 73 & c. $656 \mathrm{G}>\mathrm{T}$ & c. $1211 \mathrm{~A}>\mathrm{T}$ \\
\hline 11 & 16 & $\mathrm{~F} / 77$ & White European & NA (69) & 71 & c. $1064 A>G$ & c. $1502 \mathrm{C}>\mathrm{T}$ \\
\hline 12 & 15 & $M / 63$ & White European & Assisted & 55 & c. $1064 A>G$ & c. $1502 \mathrm{C}>\mathrm{T}$ \\
\hline 13 & 5 & $\mathrm{~F} / 9$ & White European & Independent & 20 & c. $1502 \mathrm{C}>\mathrm{T}$ & c. $1502 \mathrm{C}>\mathrm{T}$ \\
\hline 14 & 9 & $\mathrm{M} / 17$ & White European & NA (13) & 93 & c.570_571delCA & c. $980 \mathrm{C}>\mathrm{T}$ \\
\hline 15 & 5 & $\mathrm{~F} / 24$ & White European & NA (20) & 72 & c.570_571delCA & $c .980 \mathrm{C}>\mathrm{T}$ \\
\hline 16 & 18 & $\mathrm{M} / 20$ & White European & Independent & 24 & c. $1583 \mathrm{C}>\mathrm{T}$ & $\begin{array}{c}\text { c. }\left(628+1 \_6291\right) \\
\left(1536+1 \_1537-1\right) \text { del }\end{array}$ \\
\hline 17 & 14 & $\mathrm{~F} / 23$ & White European & Independent & 43 & c. $1583 \mathrm{C}>\mathrm{T}$ & $\begin{array}{c}\text { C.(628+1_6291) } \\
\left(1536+1 \_1537-1\right) \text { del }\end{array}$ \\
\hline 18 & 16 & $\mathrm{M} / 22$ & White European & Independent & 36 & c. $1583 \mathrm{C}>\mathrm{T}$ & C. $1424 \mathrm{~T}>\mathrm{C}$ \\
\hline 19 & 6 & $\mathrm{M} / 49$ & White European & Assisted & 86 & c. $401 A>G$ & c. $401 A>G$ \\
\hline 20 & 0,75 & $\mathrm{M} / 7$ & White European & NA (5) & 71 & c.570_571delCA & c. $1561 \mathrm{G}>\mathrm{A}$ \\
\hline 21 & 5 & $\mathrm{M} / 15$ & Caribbean & NA (9) & 74 & c. $680 A>G$ & c. $680 A>G$ \\
\hline 22 & 14 & $\mathrm{~F} / 31$ & White European & NA (21) & 78 & c. $1211 \mathrm{~A}>\mathrm{T}$ & c. $1561 \mathrm{G}>\mathrm{A}$ \\
\hline 23 & 10 & $\mathrm{~F} / 51$ & White European & NA (26) & 93 & c. $1561 \mathrm{G}>\mathrm{A}$ & c. $1070 \mathrm{G}>\mathrm{C}$ \\
\hline 24 & 18 & $\mathrm{M} / 31$ & White European & Independent & 23 & c. $698 \mathrm{~T}>\mathrm{A}$ & c. $1211 \mathrm{~A}>\mathrm{T}$ \\
\hline 25 & 10 & $\mathrm{M} / 18$ & Caribbean & NA (12) & 85 & c. $680 A>G$ & c. $680 A>G$ \\
\hline 26 & 16 & $\mathrm{~F} / 31$ & White European & Independent & 41 & c. $1475 C>G$ & $c .1583 C>T$ \\
\hline 27 & 12 & $\mathrm{M} / 37$ & Roma Population & Assisted & 62 & c. $1583 \mathrm{C}>\mathrm{T}$ & c. $1583 \mathrm{C}>\mathrm{T}$ \\
\hline 28 & 1,2 & $\mathrm{~F} / 18$ & White European & NA (6) & 103 & c. $1663-11 T>G$ & c. $1663-11 T>G$ \\
\hline 29 & 51 & $\mathrm{~F} / 53$ & Roma Population & NA (53) & 42 & c. $1583 \mathrm{C}>\mathrm{T}$ & c. $1583 C>T$ \\
\hline 30 & 8 & $\mathrm{~F} / 10$ & Indian & NA (2) & 86 & c. $1424 \mathrm{~T}>\mathrm{G}$ & c. $1424 T>G$ \\
\hline 31 & 3 & $\mathrm{M} / 11$ & Indian & NA (6) & 106 & c. $971 A>G$ & c. $971 A>G$ \\
\hline 32 & 1,5 & $\mathrm{M} / 11$ & White European & NA (7) & 95 & c.570_571delCA & c.570_571delCA \\
\hline 33 & 3,5 & $\mathrm{M} / 15$ & White European & NA (6) & 90 & c.570_571delCA & c.570_571delCA \\
\hline 34 & 9 & $\mathrm{~F} / 24$ & White European & Assisted & 59 & c. $1070 \mathrm{G}>\mathrm{C}$ & c. $1561 \mathrm{G}>\mathrm{A}$ \\
\hline 35 & 6 & $\mathrm{~F} / 31$ & White European & Independent & 59 & c. $1070 \mathrm{G}>\mathrm{C}$ & c. $1070 \mathrm{G}>\mathrm{C}$ \\
\hline 36 & 1 & $\mathrm{~F} / 15$ & White European & NA (7) & 60 & c.175_182del & c.175_182del \\
\hline 37 & 13 & $\mathrm{~F} / 41$ & White European & Independent & 54 & c. $1211 \mathrm{~A}>\mathrm{T}$ & c. $1561 \mathrm{G}>\mathrm{A}$ \\
\hline 38 & 20 & $\mathrm{~F} / 63$ & Roma Population & Independent & 48 & c. $1583 \mathrm{C}>\mathrm{T}$ & c. $1583 C>T$ \\
\hline 39 & 21 & $\mathrm{~F} / 64$ & Roma Population & Assisted & 51 & c. $1583 \mathrm{C}>\mathrm{T}$ & c. $1583 \mathrm{C}>\mathrm{T}$ \\
\hline 40 & 15 & $\mathrm{~F} / 24$ & White European & Independent & 34 & c. $1070 \mathrm{G}>\mathrm{C}$ & c. $1070 \mathrm{G}>\mathrm{C}$ \\
\hline 41 & 5 & $\mathrm{M} / 36$ & White European & Independent & 42 & c. $1070 \mathrm{G}>\mathrm{C}$ & c. $1070 \mathrm{G}>\mathrm{C}$ \\
\hline 42 & 20 & $\mathrm{~F} / 42$ & White European & Independent & 48 & c. $1070 \mathrm{G}>\mathrm{C}$ & c. $1070 \mathrm{G}>\mathrm{C}$ \\
\hline 43 & 11 & $\mathrm{~F} / 42$ & White European & Independent & 38 & c. $1070 \mathrm{G}>\mathrm{C}$ & c. $1070 \mathrm{G}>\mathrm{C}$ \\
\hline 44 & 1 & $\mathrm{M} / 6$ & White European & NA (6) & 67 & c.570 571delCA & c.570 571delCA \\
\hline 45 & 12 & $\mathrm{~F} / 36$ & White European & Independent & 51 & c. $1211 \mathrm{~A}>\mathrm{T}$ & c. $1561 \mathrm{G}>\mathrm{A}$ \\
\hline 46 & 30 & $\mathrm{~F} / 55$ & White European & Independent & 74 & c. $1442 G>A$ & c. $1561 \mathrm{G}>\mathrm{A}$ \\
\hline 47 & 22 & $\mathrm{M} / 25$ & Roma Population & Independent & 42 & c. $1583 \mathrm{C}>\mathrm{T}$ & c. $1583 C>T$ \\
\hline
\end{tabular}

Age is expressed in years.

F, female; Gait, age at gait loss; M, male; NA, nonambulant; NR, not reported.

severe dystonia, and according to our statement on motor examination, they were scored 4 (eg, 5 of 8 patients on finger tapping, 7 of 13 patients on leg agility, and 15 of 26 patients on postural stability). We were unable to rate freezing severity in 20 patients because they had lost gait. In addition, 3 pediatric patients (P20, P28, and P32) could not be rated on finger tapping and leg agility because of poor collaboration and cognitive impairment.

All patients showed some dystonic features. Jaw and tongue dystonia was a prominent sign $(\mathrm{n}=39 ; 83 \%)$, and it was characterized by jaw-opening dystonia $(\mathrm{n}=17 ; 36 \%)$, jaw-clenching dystonia $(\mathrm{n}=11$; $23 \%)$, and tongue protrusion $(\mathrm{n}=8 ; 17 \%)$. 
TABLE 2. Thirty-four items of the PKAN-DRS grouped in 6 subscales (SCs) and the interquartile range (IR), median, and mean \pm SD (standard deviation) values of the 47 PKAN patients included in the study

\begin{tabular}{|c|c|c|c|c|c|}
\hline \multirow[b]{2}{*}{ PKAN-DRS } & \multirow[b]{2}{*}{ Score } & \multirow[b]{2}{*}{ Patients assessed, $\mathrm{n}$} & \multicolumn{3}{|c|}{ Patient score } \\
\hline & & & IR & Median & Mean \pm SD \\
\hline \multicolumn{6}{|l|}{ SC I. Cognition } \\
\hline 1. Cognitive impairment & $0-4$ & 47 & $0-3$ & 1 & $1.4 \pm 1.5$ \\
\hline \multicolumn{6}{|l|}{ SC II. Behavior } \\
\hline 2. Psychiatric symptoms & $0-4$ & 47 & $0-3$ & 1 & $1.5 \pm 1.3$ \\
\hline \multicolumn{6}{|l|}{ SC III. Disability } \\
\hline 3. 1. Speech & $0-4$ & 47 & $2-4$ & 3 & $3 \pm 1$ \\
\hline 3.2. Handwriting & $0-4$ & 47 & $1-3$ & 2 & $2.3 \pm 1.2$ \\
\hline 3.3. Feeding & $0-4$ & 47 & $2-3$ & 2 & $2.4 \pm 1.2$ \\
\hline 3.4. Eating/swallowing & $0-4$ & 47 & $0-3$ & 2 & $1.9 \pm 1.4$ \\
\hline 3.5. Hygiene & $0-4$ & 47 & $1-4$ & 3 & $2.3 \pm 1.5$ \\
\hline 3.6. Dressing & $0-4$ & 47 & $1-4$ & 3 & $2.3 \pm 1.5$ \\
\hline 3.7. Walking & $0-5$ & 47 & $2-5$ & 4 & $3.4 \pm 1.4$ \\
\hline 3.8. School/work & $0-3$ & 47 & $2-3$ & 2 & $2.2 \pm 1$ \\
\hline 3.9. Vision & $0-3$ & 47 & $0-0$ & 0 & $0.2 \pm 0.6$ \\
\hline Total subscale & $0-35$ & & $13-27$ & 20 & $20 \pm 8.3$ \\
\hline \multicolumn{6}{|l|}{ SC IV. Parkinsonism } \\
\hline 4.1. Global spontaneity of movement (body bradykinesia) & $0-4$ & 47 & $0-3$ & 2 & $1.9 \pm 1.4$ \\
\hline 4.2. Rigidity & $0-4$ & 46 & $0-2$ & 1 & $1 \pm 1.2$ \\
\hline 4.3. Finger tapping & $0-4$ & 42 & $2-3$ & 3 & $2.5 \pm 1.2$ \\
\hline 4.4. Leg agility & $0-4$ & 43 & $1-4$ & 2,5 & $2.3 \pm 1.4$ \\
\hline 4.5 Freezing of gait & $0-4$ & 28 & $0-2$ & 0 & $1 \pm 1.3$ \\
\hline 4.6. Postural and/or trunk stability & $0-4$ & 44 & $2-4$ & 4 & $3 \pm 1.4$ \\
\hline 4.7. Resting tremor amplitude & $0-4$ & 47 & $0-1$ & 0 & $0.5 \pm 1$ \\
\hline Total subscale & $0-28$ & & $6-17$ & 12 & $11.6 \pm 6$ \\
\hline \multicolumn{6}{|l|}{ SC V. Dystonia } \\
\hline 5.1. Upper face & $0-4$ & 46 & $0-2$ & 1 & $1.2 \pm 1.2$ \\
\hline 5.2. Eye deviation & $0-4$ & 43 & $0-0$ & 0 & $0.5 \pm 1.1$ \\
\hline 5.3. Lower face & $0-4$ & 46 & $1,75-3$ & 3 & $2.5 \pm 1.2$ \\
\hline 5.4. Jaw and tongue & $0-4$ & 45 & $2-4$ & 3 & $2.6 \pm 1.3$ \\
\hline 5.5. Neck & $0-4$ & 46 & 0,3 & 2 & $1.9 \pm 1.4$ \\
\hline 5.6. Left arm & $0-4$ & 46 & $2-3$ & 3 & $2.6 \pm 1$ \\
\hline 5.7. Right arm & $0-4$ & 46 & $2-3,25$ & 2,5 & $2.6 \pm 1$ \\
\hline 5.8. Trunk & $0-4$ & 46 & 0,3 & 2 & $1.8 \pm 1.5$ \\
\hline 5.9. Left leg & $0-4$ & 46 & $2-4$ & 2 & $2.5 \pm 1.2$ \\
\hline 5.10. Right leg & $0-4$ & 46 & $1-4$ & 2 & $2.4 \pm 1.2$ \\
\hline Total subscore & $0-40$ & & $12-27$ & 19 & $20 \pm 9.5$ \\
\hline \multicolumn{6}{|l|}{ SC Vl. Other neurologic signs } \\
\hline 6.1. Speech & $0-4$ & 47 & $2-4$ & 3 & $3 \pm 1$ \\
\hline 6.2. Gait & $0-4$ & 47 & $2-4$ & 3 & $2.8 \pm 1.2$ \\
\hline 6.3. Chorea & $0-4$ & 47 & $0-0$ & 0 & $0.1 \pm 0.5$ \\
\hline 6.4. Spasticity & $0-4$ & 47 & $0-1$ & 0 & $0.5 \pm 1$ \\
\hline 6.5. Postural/action tremor & $0-4$ & 47 & $0-1$ & 0 & $0.5 \pm 0.8$ \\
\hline 6.6. Oculomotor dysfunction & $0-4$ & 40 & $0-2$ & 1 & $1 \pm 1$ \\
\hline Total subscore & $0-24$ & & $6-10$ & 8 & $7.8 \pm 2.9$ \\
\hline Total score & $0-135$ & & $43-78$ & 63 & $62 \pm 21.7$ \\
\hline
\end{tabular}

Twenty-six patients $(55 \%)$ could not walk at all (score, $4 ; \mathrm{n}=20$ ), or they needed a walking device or another person's assistance (score, $3 ; \mathrm{n}=6$ ). A significant positive correlation was observed between gait impairment and postural stability $(r=0.80)$ and lower limb dystonia $(r=0.72)$. When considering only patients who could walk, gait impairment positively correlated with the severity of freezing $(r=$ 0.66). Spasticity did not show statistical correlation with gait impairment in the whole cohort of patients.
A total of 29 patients $(62 \%)$ had at least 1 alteration in ocular motility: hypometric and slowed vertical $(\mathrm{n}=21 ; 44 \%)$ and/or horizontal saccades $(\mathrm{n}=6$; $13 \%)$, broken-up smooth pursuit $(\mathrm{n}=7 ; 15 \%)$, strabismus ( $\mathrm{n}=6 ; 12 \%)$, and gaze-evoked nystagmus $(\mathrm{n}=5 ; 10 \%)$. We were unable to rate ocular movements in 7 patients $(15 \%)$ because of a combination of severe blepharospasm $(\mathrm{n}=3)$, dystonic eye deviation $(\mathrm{n}=3)$, poor visual acuity $(\mathrm{n}=2)$, and lack of cooperation and cognitive deficit $(\mathrm{n}=2)$. 


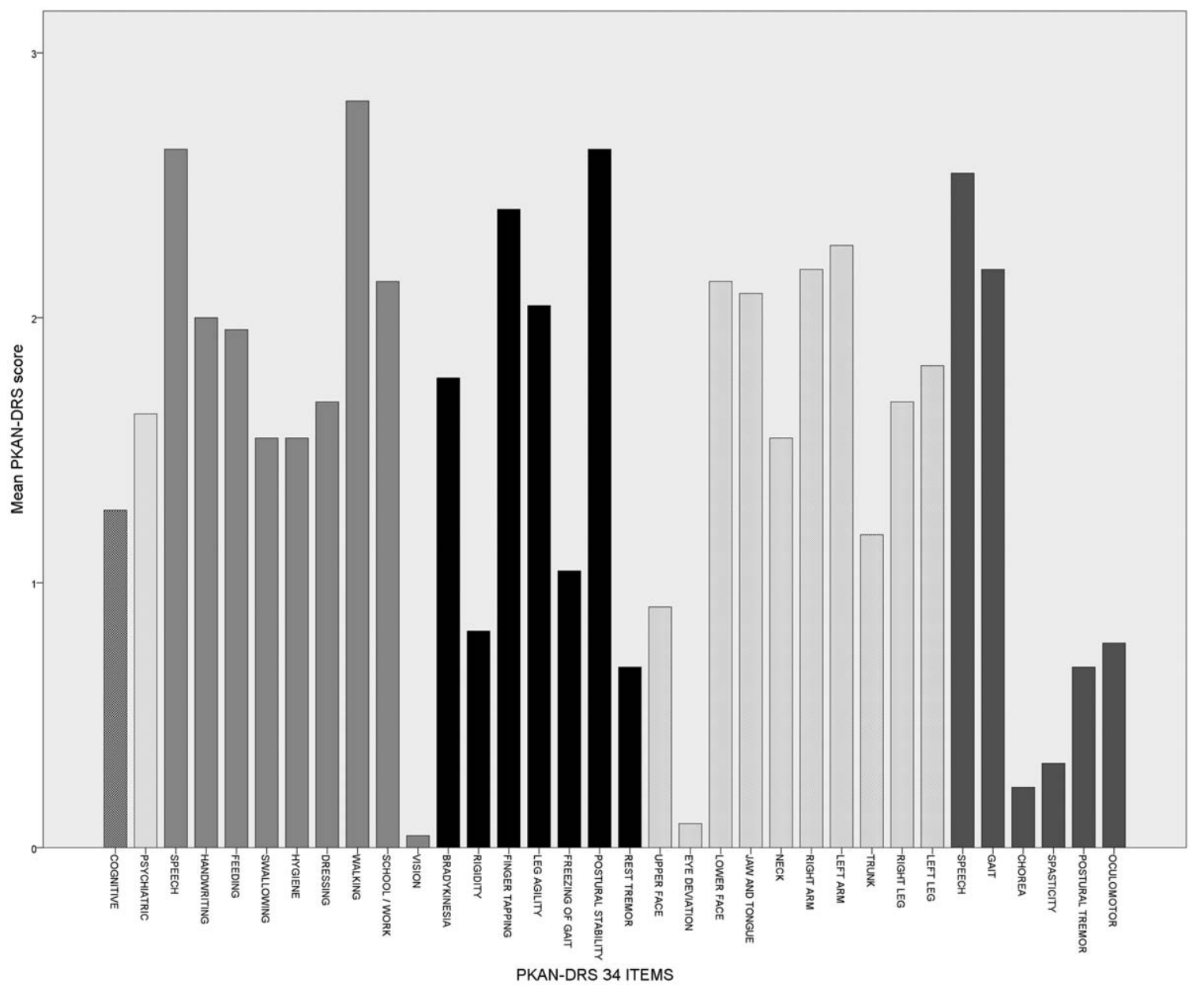

FIG. 1. Mean scores for the 34 items included in the PKAN-DRS in the cohort of 47 PKAN patients.

\section{Molecular Analysis of the PANK2 Gene}

Twenty-three different mutations were detected, 11 of which were novel (Table 1, Supplementary Figure 1, http://espinos.cipf.es/index.php/en/mutations-db). Ten Gypsy patients from Spain and Portugal were homozygous for the c.1583C $>\mathrm{T}$ (p.T528M) mutation.

\section{Clinimetric Analysis of PKAN-DRS Acceptability}

SCs I, II, III, and VI showed fully computable data. SCs IV and V presented $2.1 \%$ and $2.9 \%$ of missing data, respectively. We observed a mild/moderate floor effect in SCs I and II. The complete analysis is shown in Supplementary Table 1.

\section{Internal Consistency}

A high level of internal consistency was observed for the total scale $(\alpha=0.87)$. For the 4 subscales, the values were: SC III, $\alpha=0.89$; SC IV, $\alpha=0.85$; SC V, $\alpha$ $=0.91 ;$ and SC VI, $\alpha=0.33$.
Corrected item-total correlations for SCs III (range, 0.40-0.87), IV (range, 0.39-0.74), V (range, 0.350.83 ), and VI (range, -0.09-0.37) are shown in Supplementary Table 2 .

Correlations among the PKAN-DRS I-VI SCs are stated in Supplementary Table 3.

\section{Interrater Reliability}

Table 3 summarizes the $\mathrm{k}_{\mathrm{w}}$ values for the individual items (mean $\mathrm{k}_{\mathrm{w}}, 0.30-0.93$ ). Substantial or excellent agreement was achieved in 18 of 21 items $(85 \%)$. The oculomotor assessment and left arm dystonia showed moderate agreement, whereas dystonia of the lower face showed fair agreement. Supplementary Table 4 shows the ICCs for interobserver agreement for the total scale and SCs IV-VI.

\section{Convergent Validity}

A total of 21 patients were evaluated by rater 2 using the BFM-DRS. In these patients, the correlation between the BFM-DRS and the PKAN-DRS was 0.93 $(P<0.001)$. 
TABLE 3. Analysis of interrater agreement: weighted kappa for individual items in 28 patients tested

\begin{tabular}{|c|c|c|c|c|}
\hline Item & $\begin{array}{c}\text { к (w) } \\
\text { Rater 2-1 }\end{array}$ & $\begin{array}{c}\text { к (w) } \\
\text { Rater 2-3 }\end{array}$ & $\begin{array}{c}\text { к (w) } \\
\text { Rater 1-3 }\end{array}$ & $\begin{array}{c}\text { Mean } \\
\kappa(w)\end{array}$ \\
\hline Speech & 0.85 & 0.88 & 0.88 & 0.87 \\
\hline Gait & 0.94 & 0.93 & 0.93 & 0.93 \\
\hline Chorea & 0.77 & 0.71 & 0.64 & 0.70 \\
\hline Postural tremor & 0.90 & 0.55 & 0.72 & 0.72 \\
\hline Oculomotor & 0.67 & 0.32 & 0.41 & 0.47 \\
\hline Bradykinesia & 0.80 & 0.75 & 0.79 & 0.78 \\
\hline Finger tapping & 0.83 & 0.76 & 0.74 & 0.78 \\
\hline Heel stomping & 0.80 & 0.71 & 0.62 & 0.71 \\
\hline Freezing & 0.93 & 0.81 & 0.86 & 0.87 \\
\hline Postural instability & 0.97 & 0.83 & 0.80 & 0.87 \\
\hline Resting tremor & 0.94 & 0.54 & 0.59 & 0.69 \\
\hline Upper face & 0.77 & 0.71 & 0.78 & 0.75 \\
\hline Eye deviation & 0.63 & 0.54 & 0.71 & 0.63 \\
\hline Lower face & 0.83 & 0.03 & 0.03 & 0.30 \\
\hline Jaw and tongue & 0.89 & 0.64 & 0.78 & 0.77 \\
\hline Neck & 0.88 & 0.67 & 0.72 & 0.76 \\
\hline Left arm & 0.72 & 0.45 & 0.61 & 0.59 \\
\hline Right arm & 0.76 & 0.71 & 0.77 & 0.75 \\
\hline Trunk & 0.88 & 0.70 & 0.59 & 0.72 \\
\hline Left leg & 0.87 & 0.71 & 0.76 & 0.78 \\
\hline Right leg & 0.87 & 0.70 & 0.68 & 0.75 \\
\hline
\end{tabular}

к (w), weighted kappa.

\section{Known-Groups Validity}

The PKAN-DRS showed the ability to discriminate between 2 groups of patients according to the genotype. Ten gypsy patients homozygous for the c.1583C $>\mathrm{T}$ (p. T528M) mutation showed lower values for the PKAN-DRS total score and for SCs V and VI than patients with other mutations (Fig. 2). Homozygous c.1583C $>\mathrm{T}$ patients had a later age at disease onset (19 \pm 12 years; range, 6-51 years) than patients with other mutations $(10 \pm 8$ years; $1-34$ years; $P=0.004)$. No differences between the groups were observed in the duration of the disease.

\section{Additional Results}

We did not observe differences between the sexes in the scores on the scale. We performed linear regression analysis including PKAN-DRS total scale and SCs as dependent variables and age at disease onset and years of disease duration as independent variables. This analysis showed a significant negative correlation between age at onset and SC III (beta $=-0.43, P=0.003$ ), SCI V (beta $=-0.66, P=0.004)$ and SC VI (beta $=-0.29, P=$ 0.047). In addition, this analysis showed a significant positive association between years of disease evolution and SC IV (beta $=0.60, P<0.0001$ ).

\section{Discussion}

The PKAN-DRS was designed to include all motor and nonmotor aspects of PKAN and the consequent functional impairment across different populations. Forty-seven patients aged 6 to 77 years were examined at the referral centers in collaboration with the different investigators. This methodology reduced the substantial burden of patients and caregivers, ensured

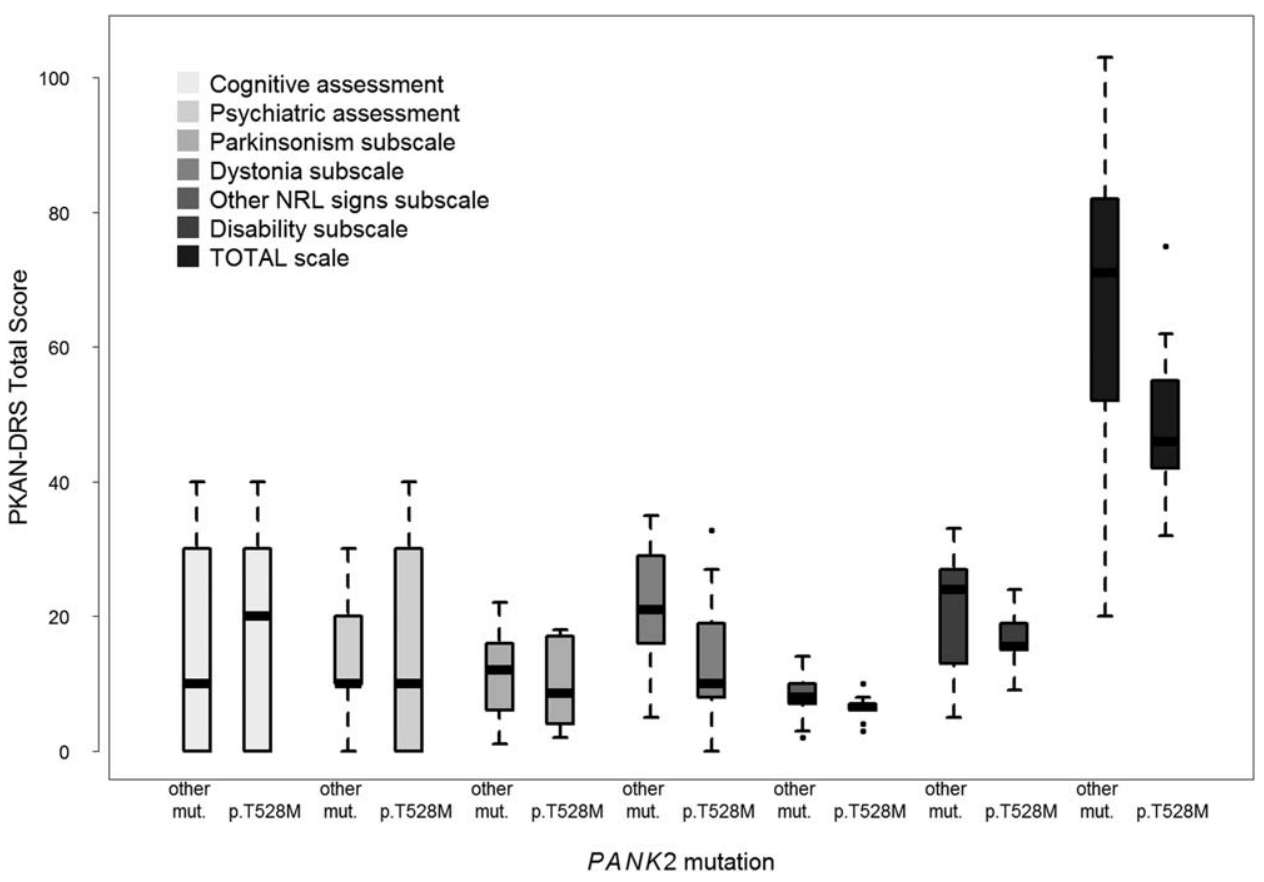

FIG. 2. Graphic representation of patients harboring the c.1583C>T (p.T528M) mutation in homozygosis compared with patients with other mutations. Total score, $P=0.02$; SC I (cognition), $P=0.55$; SC II (behavior), $P=0.93$; SC III (disability), $P=0.09$; SC IV (parkinsonism), $P=0.40$; SC V (dystonia), $P=0.01$; SC VI (other neurologic signs), $P=0.04$ (Mann-Whitney $U$ test). The values of SC I and SC II have been multiplied by 10 to show comparable blog spots. 
success in the recruitment of patients, and allowed the uniform collection of data and a systematic and standardized evaluation of patients. Moreover, as telemedicine has been considered a reliable tool in clinical research of rare diseases, ${ }^{31}$ we developed a videorecording protocol for the motor examination that allowed the assessment of patients by different raters for clinimetric analysis.

Dystonia and parkinsonism, as core features of PKAN, had a relevant representation in the global scale. We quantified dystonia in a standard setting that allowed for the identification of the maximal severity in the same manner in all patients. Dystonia was present in all 47 patients with predominant involvement of the trunk, jaw and tongue, causing retrocollis, jaw opening, jaw clenching, or tongue protrusion. ${ }^{32-34}$ Moreover, the severity of dystonia prevented essential daily activities in a relevant subgroup of patients. One third of patients also showed a prominent action-induced blepharospasm that interfered with the assessment of ocular motility in some cases.

We identified parkinsonian features in all patients with a highly variable range of severity. Freezing of gait was a common feature, present in $48 \%$ of patients with assisted or independent gait. Similar to other series, resting tremor was observed in a minority of cases $(15 \%){ }^{2}$ This is in contrast to Parkinson's disease, in which resting tremor emerges in the majority of patients. ${ }^{35}$

Disability, dystonia, and other neurological signs were more severe in patients with earlier onset of disease, whereas parkinsonian features were more common in older patients with longer disease duration. Longitudinal studies will be necessary to confirm these results and to determine the existence of a specific phenotype according to the age of presentation.

Ocular dysmotility, especially hypometric and slowed vertical saccades, was a common sign in our series. A dysfunction of the key structures that control conjugate eye movements in the midbrain and the pons may account for these findings in PKAN. ${ }^{36,37}$

Regarding disability, more than half our PKAN population either could not walk at all or needed a walking device or another person's assistance. We found that the main contributors to gait impairment were postural stability and lower limb dystonia. Freezing of gait was also a relevant factor in ambulant patients. Dysarthria and dysphagia impaired or prevented speech and swallowing in most patients, ${ }^{32,33,38}$ probably resulting from a combination of hyper- or hypokinesia in the orofacial, pharyngeal, and laryngeal muscles. The disease also showed a strong impact on school attendance and academic abilities in childhood and later prevented patients from engaging in gainful employment in adulthood.

Cognitive impairment and psychiatric symptoms were common features. Depression, irritable behavior, obsessions and compulsions, and disruptive or aggressive behavior predominated in our cohort, as in previous reports. ${ }^{19,37,38}$

The PKAN-DRS showed good internal consistency for the total scale and SCs. Exceptionally low values were observed for SC VI when addressing other neurological signs. Speech, chorea, spasticity, postural tremor, and ocular dysmotility showed low correlation with total SC VI. The heterogenic nature of the items included in this subscale, together with the low prevalence of some of them, particularly chorea, spasticity, and postural tremor, may account for these results. In addition, correlations among the different subscales showed that dystonia and parkinsonism were independent variables with no correlation between them. This is an important finding, as we initially observed that severe dystonia was interfering with the assessment of parkinsonian features in one-third of the sample. On the other hand, the disability subscale showed moderate correlation with the parkinsonism subscale and strong correlation with the subscales rating dystonia and other neurological disturbances, confirming that these aspects had a significant impact on a patient's capability of performing activities of daily living.

Despite the wide range of ages included in this study and the different stages of evolution, most PKAN patients could follow the instructions to perform the different items of the scale. We recorded missing data of less than $4 \%$, demonstrating good acceptability of the scale. Based on the statistical analysis, we assume that the PKAN-DRS satisfied the criteria for acceptability. However, SCs I and II showed a mild/moderate floor effect, reflecting the proportion of patients without manifestations in these domains.

In this initial validation study, substantial or excellent interrater reliability was demonstrated in most items. Fair agreement was found in the assessment of lower face dystonia, which may be difficult to differentiate from oromandibular dystonia, a prominent sign in PKAN. Finally, we observed a good correlation between the PKAN-DRS and the BFM-DRS, which allowed us to establish convergent validity for the PKAN-DRS and to compare our findings in the context of the existing literature.

The missense mutation c. $1583 \mathrm{C}>\mathrm{T}$ (p.T528M) is one of the most frequent mutations observed in the PANK2 gene. ${ }^{19,39-43}$ In our series, all the Gypsy patients harbored the PANK2 c. $1583 \mathrm{C}>\mathrm{T}$ change in homozygosis, suggesting a founder effect for this mutation in the Gypsy population from the Iberian Peninsula. These patients had a more benign phenotype, with lower scores for the total PKAN-DRS scale and subscales. This analysis supported construct validity for the scale, as it discriminated a specific genotype with milder severity.

This study also has some limitations. Regarding scale validation, the sample size is under the minimal 
number usually accepted for most basic clinimetric attributes. ${ }^{44}$ However, given the rarity of the condition that hinders the recruitment of a sufficient sample of patients, we succeeded in recruiting 47 patients, which represented a clinically reasonable number of cases for this rare condition. ${ }^{19,32-34,37,38}$ According to the acceptability analysis, the items for cognitive and psychiatric evaluation should be refined to improve the sensitivity of the assessment, and cognitive pretesting methods may be necessary in the future to assess these domains. The inclusion of items with very low prevalence in our series can be questioned. The next steps will involve the assessment of a larger series of PKAN patients to help us decide if these items should be removed or should remain to ensure the multidimensional quality of the scale. In addition, as a word of caution, the PKAN-DRS focuses on neurological disturbances that contribute significantly to functional disability, but it should not be forgotten that other scales of well-being, activity, and participation will be required for any balanced intervention in this complex and progressive disorder. ${ }^{45}$ Nevertheless, a mark of disease stability, that is, not improved but not worse, can also be a "measure" of success, and the PKANDRS may play an important role for monitoring these patients.

In conclusion, in this pilot study, we demonstrated that the proposed PKAN-DRS seems to be a reliable and valid scale for assessment of PKAN patients. It allowed an accurate assessment of severity in a cohort of patients who included children, adolescents, adults, and the elderly. We demonstrated that the earlier the onset of the disease, the higher are the ratings for disability, dystonia, and other neurological signs. Also, dystonia and other neurological signs had a strong impact on functional capability. Finally, we identified a strong phenotype-genotype correlation in a cohort of homozygous c.1583C $>\mathrm{T}$ Gypsy patients with a milder phenotype. In the future, the development of a teaching videotape will facilitate interrater reliability. Also, additional validation studies with a larger sample size will be required to confirm the present results and to complete the scale validation testing. Hopefully, the PKAN-DRS will be a useful tool in both clinical practice and research.

Acknowledgments: We thank the patients and their families for agreeing to participate in this study.

\section{References}

1. Gregory A, Polster BJ, Hayflick SJ. Clinical and genetic delineation of neurodegeneration with brain iron accumulation. J Med Genet 2009;46:73-80.

2. Hogarth P. Neurodegeneration with brain iron accumulation: diagnosis and management. J Mov Disord 2015;8:1-13.

3. Burke R, Fhan S, Marsden CD, et al. Validity and reliability of a rating scale for primary torsion dystonias. Neurology 1985;35:7377.
4. Fahn S, Elton RL, UPDRS Program Members. Unified Parkinson's disease rating scale. In: Fahn S, Marsden CD, Goldstein M, Calne DB, eds. Recent Developments in Parkinson's Disease, Vol. 2. Florham Park, NJ: Macmillan Healthcare Information; 1987:153-163, 293-304.

5. Trouillas P, Takayanagi T, Hallett M, et al. International Cooperative Ataxia Rating Scale for pharmacological assessment of the cerebellar syndrome. The Ataxia Neuropharmacology Committee of the World Federation of Neurology. J Neurol Sci 1997;145:205211.

6. Comella CL, Leurgans S, Wuu J, Stebbins GT, Chmura T; Dystonia Study Group. Rating scales for dystonia: a multicenter assessment. Mov Disord 2003;18:303-312.

7. Krause M, Wolfgang F, Tronnier V, et al. Long-Term Benefit to Pallidal Deep Brain Stimulation in a Case of Dystonia Secondary to Pantothenate Kinase-Associated Neurodegeneration. Mov Disord 2006;21:2255-2257.

8. Timmermann L, Pauls KA, Wieland K, et al. Dystonia in neurodegeneration with brain iron accumulation: outcome of bilateral pallidal stimulation. Brain 2010;133:701-712.

9. Adamovicová M, Jech R, Urgošík D, Spacková N, Krepelová A. Pallidal stimulation in siblings with pantothenate kinase-associated neurodegeneration: four-year follow-up. Mov Disord 2011;26:184187.

10. Lumsden DE, Kaminska M, Gimeno H, et al. Proportion of life lived with dystonia inversely correlates with response to pallidal deep brain stimulation in both primary and secondary childhood dystonia. Dev Med Child Neurol 2013;55:567-574.

11. Abbruzzese G, Cossu G, Balocco M, et al. A pilot trial of deferiprone for neurodegeneration with brain iron accumulation. Haematologica 2011;96:1708-1711.

12. Zorzi G, Zibordi F, Chiapparini L, et al. Iron-related MRI images in patients with pantothenate kinase-associated neurodegeneration (PKAN) treated with deferiprone: results of a phase II pilot trial. Mov Disord 2011;26:1756-759.

13. Cossu G, Abbruzzese G, Matta G, et al. Efficacy and safety of deferiprone for the treatment of pantothenate kinase-associated neurodegeneration (PKAN) and neurodegeneration with brain iron accumulation (NBIA): results from a four years follow-up. Parkinsonism Relat Disord 2014;20:651-654.

14. Schneider SA, Bhatia KP, Hardy J. Complicated recessive dystonia parkinsonism syndromes. Mov Disord 2009;24:490-499.

15. Huntington Study Group. Unified Huntington's Disease Rating Scale: reliability and consistency. Mov Disord 1996;11:136-142.

16. Goetz CG, Tilley BC, Shaftman SR, et al. Movement Disorder Society UPDRS Revision Task Force. Movement Disorder Societysponsored revision of the Unified Parkinson's Disease Rating Scale (MDS-UPDRS): scale presentation and clinimetric testing results. Mov Disord 2008;23:2129-2170

17. Wenning GK, Tison F, Seppi K, et al. Multiple System Atrophy Study Group. Development and validation of the Unified Multiple System Atrophy Rating Scale (UMSARS). Mov Disord 2004;19: 1391-1402.

18. Ashworth B. Preliminary trial of carisoprodal in multiple sclerosis. Practitioner 1964;192:540-542.

19. Hayflick SJ, Westaway SK, Levinson B, et al. Genetic, clinical, and radiographic delineation of Hallervorden-Spatz syndrome. N Engl J Med 2003;348:33-40.

20. Martínez-Martín P, Gil-Nagel A, Gracia LM, Gómez JB, Martínez-Sarriés J, Bermejo F. Unified Parkinson's Disease Rating Scale characteristics and structure. The Cooperative Multicentric Group. Mov Disord 1994;9:76-83.

21. Agostino R, Currà $\mathrm{A}$, Giovannelli $\mathrm{M}$, Modugno N, Manfredi M, Berardelli A. Impairment of individual finger movements in Parkinson's disease. Mov Disord. 2003;18:560-565.

22. Bologna M, Leodori G, Stirpe P, Paparella G, Colella D, Belvisi D, Fasano A, Fabbrini G, Berardelli A. Bradykinesia in early and advanced Parkinson's disease. J Neurol Sci 2016;369:286-291.

23. Albanese A, Sorbo FD, Comella C, et al. Dystonia rating scales: critique and recommendations. Mov Disord 2013;28:874-883.

24. Smith SC, Lamping DL, Banarjee S, et al. Measurement of health related quality of life for people with dementia: development of a new instrument (DEMQOL) and an evaluation of current methodology. Health Technol Assess 2005;9:16-19. 
25. Hobart JC, Riazi A, Lamping DL, Fitzpatrick R, Thompson AJ. Improving the evaluation of therapeutic interventions in multiple sclerosis: development of a patient-based measure of outcome. Health Technol Assess 2004;8:1-48.

26. van der Linden FA, Kragt JJ, Klein M, van der Ploeg HM, Polman $\mathrm{CH}$, Uitdehaag BM. Psychometric evaluation of the multiple sclerosis impact scale (MSIS-29) for proxy use. J Neurol Neurosurg Psychiatry. 2005;76:1677-1681.

27. Landis JR, Koch GG. The measurement of observer agreement for categorical data. Biometrics 1977; 33:159-174.

28. Fisk JD, Brown MG, Sketris IS, Metz LM, Murray TJ, Stadnyk KJ. A comparison of health utility measures for the evaluation of multiple sclerosis treatments. J Neurol Neurosurg Psychiatry. 2005;76:58-63.

29. Hobart J, Lamping D, Fitzpatrick R, Riazi A, Thompson A. The Multiple Sclerosis Impact Scale (MSIS-29): a new patient-based outcome measure. Brain. 2001;124:962-973.

30. Portney LG, Watkins MP. Foundations of Clinical Research: Applications to Practice. 3rd ed. Norwalk, CT: Appleton and Lange; 1993:588-595, 606-607.

31. Cialone J, Augustine EF, Newhouse N, Vierhile A, Marshall FJ, Mink JW. Quantitative telemedicine ratings in Batten disease: implications for rare disease research. Neurology 2011;77:1808-1811.

32. Assami S, Azzedine H, Nouioua S, et al. Pantothenate kinaseassociated neurodegeneration: clinical description of 10 patients and identification of new mutations. Mov Disord 2011;26:1777-1789.

33. Tomić A, Petrović I, Svetel M, Dobričić V, Dragašević Mišković N, Kostić VS. Pattern of disease progression in atypical form of pantothenate-kinase-associated neurodegeneration (PKAN) - Prospective study. Parkinsonism Relat Disord 2015;21:521-524.

34. Lee JH, Park J, Ryu HS, et al. Clinical Heterogeneity of Atypical Pantothenate Kinase-Associated Neurodegeneration in Koreans. J Mov Disord 2016;9:20-27.

35. Fahn S, Jankovic J, eds. Principles and Practice of Movement Disorders, Philadelphia: Churchill Livingstone; 2007:82.

36. Egan RA, Weleber RG, Hogarth P, et al. Neuro-ophthalmologic and electroretinographic findings in pantothenate kinase-associated neurodegeneration (formerly Hallervorden-Spatz syndrome). Am J Ophthalmol 2005;140:267-274.
37. Morales-Briceño H, Chacón-Camacho OF, Pérez-González EA, et al. Clinical, imaging, and molecular findings in a sample of Mexican families with pantothenate kinase-associated neurodegeneration. Clin Genet 2015;87:259-265.

38. Pellecchia MT, Valente EM, Cif L, et al. The diverse phenotype and genotype of pantothenate kinase-associated neurodegeneration. Neurology 2005;64:1810-1812.

39. Zhou B, Westaway SK, Levinson B, Johnson MA, Gitschier J, Hayflick SJ. A novel pantothenate kinase gene (PANK2) is defective in Hallevorden-Spatz syndrome. Nat Genet 2001;28:345-349.

40. Zhang YM, Rock CO, Jackowski S. Biochemical properties of human pantothenate kinase 2 isoforms and mutations linked to pantothenatekinase-associated neurodegeneration. J Biol Chem 2006;281:107-114.

41. Hartig MB, Hörtnagel K, Garavaglia B, et al. Genotypic and phenotypic spectrum of PANK2 mutations in patients with neurodegeneration with brain iron accumulation. Ann Neurol 2006;59: 248-256.

42. Wu YW, Hess CP, Singhal NS, Groden C, Toro C. Idiopathic basal ganglia calcifications: an atypical presentation of PKAN. Pediatr Neurol 2013;49:351-354.

43. García-Ruiz PJ, Ayerbe J, Vela Desojo L, Feliz CE, Del Val Fernandez J. Deep brain stimulation for pantothenate kinaseassociated neurodegeneration. Case Rep Neurol Med 2015;2015:1 3 .

44. Terwee CB, Bot SD, de Boer MR, et al. Quality criteria were proposed for measurement properties of health status questionnaires. J Clin Epidemiol 2007;60:34-42.

45. Lumsden DE, Gimeno H, Tustin K, Kaminska M, Lin JP. Interventional studies in childhood dystonia do not address the concerns of children and their carers. Eur J Paediatr Neurol 2015;19:327-336.

\section{Supporting Data}

Additional Supporting Information may be found in the online version of this article at the publisher's website. 